\title{
Ionized and neutral gas in the XUV discs of nearby spiral galaxies
}

\author{
López-Sánchez $^{1,2}$, B. S. Koribalski ${ }^{3}$, T. Westmeier ${ }^{4}$ and C. Esteban ${ }^{5,6}$ \\ ${ }^{1}$ Australian Astronomical Observatory, PO Box 915, North Ryde, NSW 1670, Australia \\ email: Angel.Lopez-Sanchez@aao.gov.au \\ ${ }^{2}$ Department of Physics and Astronomy, Macquarie University, NSW 2109, Australia \\ ${ }^{3}$ CSIRO Astronomy and Space Science, Australia Telescope National Facility, PO BOX 76, \\ Epping, NSW 1710, Australia \\ ${ }^{4}$ International Centre for Radio Astronomy Research, The University of Western Australia, 35 \\ Stirling Hwy, Crawley, WA 6009, Australia \\ ${ }^{5}$ Instituto de Astrofísica de Canarias, E-38200 La Laguna, Tenerife, Spain \\ ${ }^{6}$ Departamento de Astrofísica, Universidad de La Laguna, E-38205 La Laguna, Tenerife, Spain
}

\begin{abstract}
We are conducting a multiwavelength study of XUV discs in nearby, gas-rich spiral galaxies combining the available UV (GALEX) observations with $\mathrm{H}$ I data obtained at the ATCA as part of the Local Volume HI Survey (LVHIS) project and multi-object fibre spectroscopy obtained using the $2 \mathrm{dF} / \mathrm{AAOmega}$ instrument at the $3.9 \mathrm{~m}$ AAT. Here we present the results of the multiwavelength analysis of the galaxy pair NGC $1512 / 1510$. The H I distribution of NGC 1512 is very extended with two pronounced spiral/tidal arms. Hundreds of independent UV-bright regions are associated with dense $\mathrm{H}$ I clouds in the galaxy outskirts. We confirm the detection of ionized gas in the majority of them and characterize their physical properties, chemical abundances and kinematics. Both the gas distribution andthe distribution of the star-forming regions are affected by gravitational interactionwith the neighbouring blue compact dwarf galaxy NGC 1510. Our multiwavelength analysis provides new clues about local star-formation processes, the metal redistribution in the outer gaseous discs of spiral galaxies, the importance of galaxy interactions, the fate of the neutral gas and the chemical evolution in nearby galaxies.
\end{abstract}

Keywords. galaxies: ISM, abundances, kinematics and dynamics, evolution, interactions, dwarf, individual: NGC 1510, NGC 1512

\section{Introduction}

The study of star-formation processes in galaxy outskirts gives key clues about the formation and evolution of galaxies, not only because these regions may probe physical conditions similar to those present of the early Universe, but also because they test the inside-out scenario of galaxy disc formation. The discovery of UV-bright complexes in the outskirts (beyond their $\mathrm{H} \alpha$ or $B_{25}$ radius) of nearby spiral galaxies (Thilker et al. 2005; Gil de Paz et al. 2005) has provided an excellent opportunity to investigate both how stars are formed in regions of low gas density and the chemical enrichment of the outer parts of galaxies. When combining the UV data with optical spectroscopy and 21-cm H I observations we get important constraints to the star-formation activity and star-formation history of galaxies, the relationships between gas and stars, as well as the physics behind them. We are conducting such study of XUV discs in nearby, H I -rich galaxies included in the Local Volume HI Survey (LVHIS) project (Koribalski 2008, Koribalski et al. in prep.) using the multi-object fiber-positioner instrument $2 \mathrm{dF}$ and the AAOmega spectrograph installed at the $3.9 \mathrm{~m}$ Anglo-Australian Telescope (AAT, Siding Spring Observatory, NSW, Australia). 


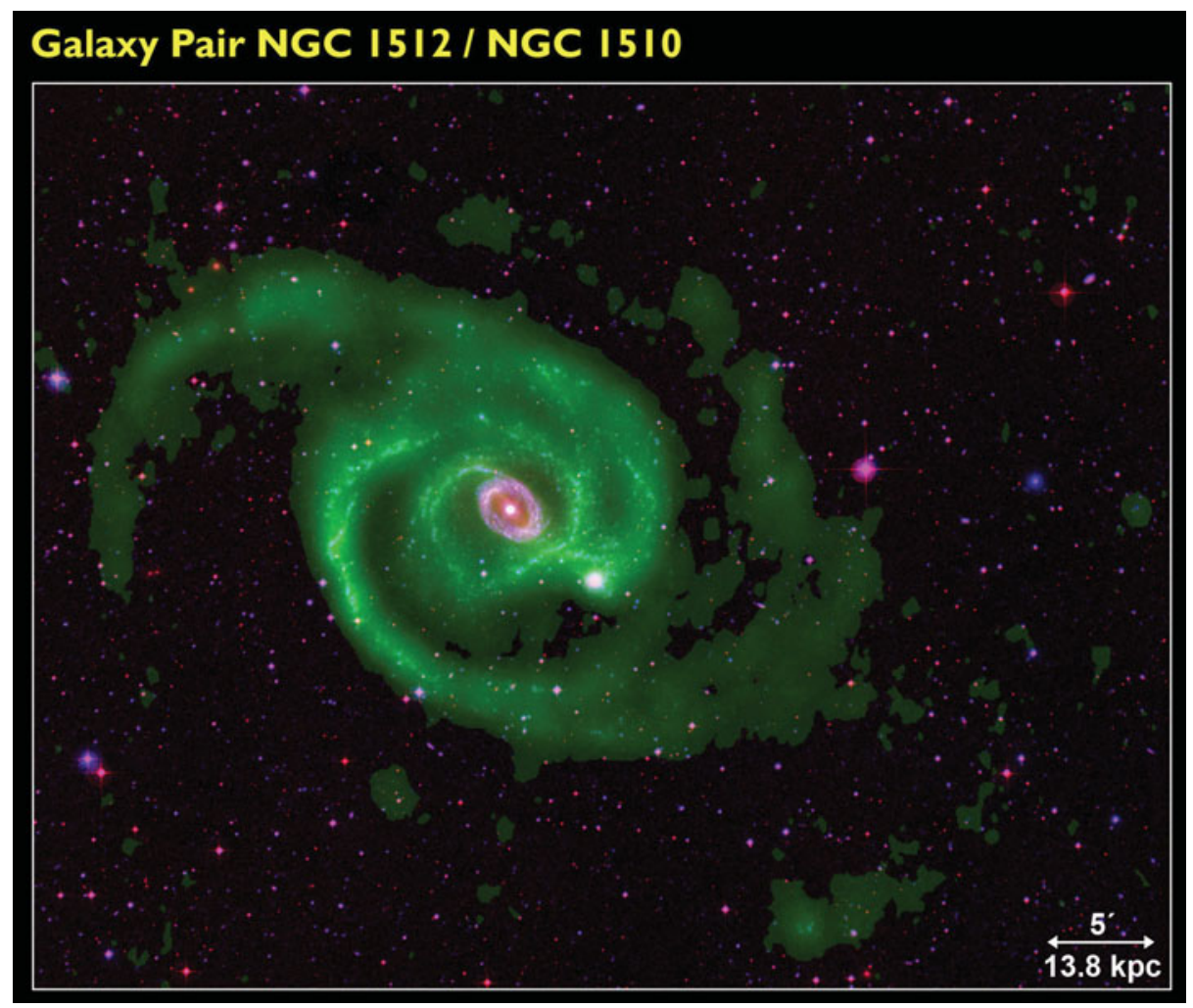

Figure 1. Multi-wavelength color-composite image of the galaxy pair NGC 1512/1510 obtained usingthe DSS $R$-band image (red), the ATCA H I distribution (green) and the GALEX NUV -band image (blue).The Spitzer $24 \mu \mathrm{m}$ image was overlaid just in the center of the two galaxies. We note that in the outer diskthe UV emission traces the regions of highest $\mathrm{H}$ I column density.

Here we present the results of our analysis of the XUV emission in the galaxy pair NGC 1512/1510. At the adopted distance of $9.5 \mathrm{Mpc}, 1^{\prime}$ corresponds to $2.49 \mathrm{kpc}$. This system hosts hundreds of independent UV-bright regions associated with dense $\mathrm{H} \mathrm{I}$ clouds in the outskirts, the blue compact dwarf galaxy (BCDG) NGC 1510 (which is located at just $13.8 \mathrm{kpc}$ from the center of NGC 1512), a central star-forming ring and two tidal dwarf galaxy (TDG) candidates at very large distance (projected radius of 83 $\mathrm{kpc}$ ) identified by their $\mathrm{H}$ I emission.

\section{Neutral gas distribution in NGC $1512 / 1510$}

The analysis of the neutral gas distribution in NGC 1512/1510 was presented in Koribalski \& López-Sánchez (2009). Our deep ATCA H I mosaic revealed a very extended $(\sim 4 \times$ its optical diameter) $\mathrm{H}$ I distribution (see Fig. 1$)$, spanning a diameter of $\sim 40$ (or $110 \mathrm{kpc}$ ). Two prominent spiral arms, which appear to wrap around $\sim 1.5$ times, are among the mostremarkable $\mathrm{H}$ I features. The brightness and width of both $\mathrm{H}$ I arms varies with radius. The southern arm splits into three branches, followed by a broad region of $\mathrm{H}$ I debris towards the west before continuing on as a single feature towards the north. Thesedisturbances in the outer disk of NGC 1512 are likely caused by tidal interaction with andaccretion of the dwarf companion, NGC 1510. Individual H I clouds are found out to projectedradii of $30(\sim 83 \mathrm{kpc})$. The velocity gradient detected within 


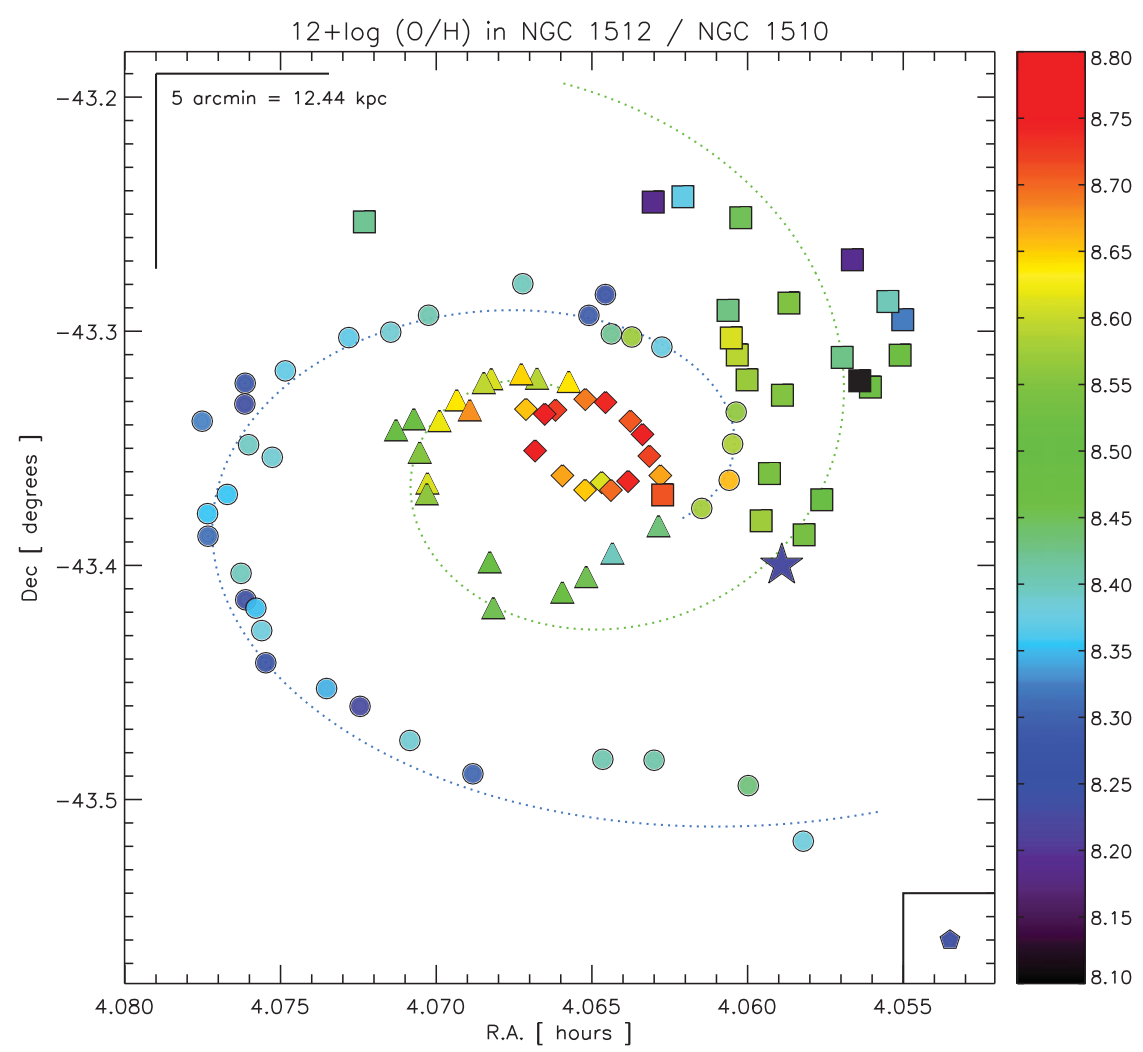

Figure 2. Map of the oxygen abundance in the NGC 1512/1510 system, as it is provided via the analysis of the emission lines detected in the UV-rich regions. The four areas identified in the system are shown using different symbols: Area $1=$ Ring (Diamonds), Area $2=$ Arm 1 (circles), Area $3=$ Internal Arm (triangles), Area $4=$ External Debris (squares). The position of the BCDG NGC 1510 is shown with a star, while the TDG 1 (which actually lies far from the system, at $78 \mathrm{kpc}$ from the center of NGC 1512) is plotted using a pentagon. The scale included in the top left corner indicates the length of $5 \mathrm{arcmin}$, which corresponds to $12.44 \mathrm{kpc}$ at the distance of NGC 1512. The value for $R_{25}$ in NGC 1512 is $11.76 \mathrm{kpc}(4.26 \operatorname{arcmin})$. The two $\mathrm{H}$ I tidal/spiral arms are sketched following a blue (Arm 1) or green (Arm 2) dotted line.

the extended clumps agrees withthat of the neighbouring spiral arms, suggesting that they are condensations within theoutermost parts of the disk. We detect star-formation activity in the two most distant $\mathrm{H}$ I clumps (TDG candidates). The large majority of the observed UV-complexes lie in regions where the $\mathrm{H}$ I column density isabove $2 \times 10^{21}$ atoms $\mathrm{cm}^{-2}$. Hence, XUV-discs should be embedded in larger $\mathrm{H}$ I envelopes $-\mathrm{a} 2 \mathrm{X}-\mathrm{H}$ I disc--, which are providing the fuel for their star-formation activity.

\section{Ionized gas in the XUV regions of NGC 1512/1510}

Our 2dF/AAOmega observations (López-Sánchez et al. in revision) provided deep, intermediate-resolution optical spectroscopy of 136 genuine UV-bright regions located in both the spiral and the XUV discs of the NGC 1512/1510 system. We detect ionized gas in the huge majority of them and confirm that in all cases except the centre of NGC 1512 photoionization by massive stars is the main excitation mechanism of the gas. In the XUV complexes, only few (1-5) O7V stars are responsible for this. 
Using a comprehensive analysis of the emission lines of [ $\mathrm{O}$ II ] $\lambda 3727, \mathrm{H} \gamma, \mathrm{H} \beta$, [ O III ] $\lambda 5007, \mathrm{H} \alpha$, and [ N II ] $\lambda 6583$ we computed the oxygen abundance and the N/O ratio for the majority of the detected UV-bright regions. The obtained chemical abundance map (see Fig. 2) reaches a projected distance of $78 \mathrm{kpc}\left(6.6 R_{25}\right)$ from the center of NGC 1512. We found significant differences between regions along the Arm 1 at the east, which have oxygen abundances $8.25 \lesssim 12+\log (\mathrm{O} / \mathrm{H}) \lesssim 8.45$, and knots located in the external debris of Arm 2 , which typically have $8.40 \lesssim 12+\log (\mathrm{O} / \mathrm{H}) \lesssim 8.60$. Considering just a radial gradient involving all complexes it is difficult to see a radial decrease of metallicity from the center of NGC 1512 to the outer regions. However, assuming both a radial and an azimuthal gradient following the spiral arms we are able to clearly distinguish that Arm 1 has an almost flat oxygen abundance $-12+\log (\mathrm{O} / \mathrm{H}) \sim 8.35-$ and flat $\mathrm{N} / \mathrm{O}$ ratio $-\log (\mathrm{N} / \mathrm{O}) \sim$ -1.4- while regions located in the disrupted Arm 2 (inner arm and external debris), show a large dispersion in oxygen abundances $-8.1 \lesssim 12+\log (\mathrm{O} / \mathrm{H}) \lesssim 8.6-$ and N/O ratios. Arm 2 has experienced an enhancement in star-formation because of the interaction with NGC 1510, flattening the radial metallicity at large radii, while Arm 1 still shows the original and poorly-disturbed radial distribution (gradient+flat in the outskirts).

Using the $\mathrm{H} \alpha$ emission line profile we trace the kinematics of the system out to $78 \mathrm{kpc}$, which generally matches well with that provided by the $\mathrm{H}$ I kinematics (velocity differences of less than $15 \mathrm{~km} \mathrm{~s}^{-1}$ ). Some small velocity discrepancies (differences between 20 and $40 \mathrm{~km} \mathrm{~s}^{-1}$ ) are found in some clusters of the external debris and in particular areas of Arm 1. We locate a region in the internal arm for which its $\mathrm{H} \alpha$ radial velocity differs by $136 \mathrm{~km} \mathrm{~s}^{-1}$ with respect its $\mathrm{H}$ I radial velocity. The upper limit to the metallicity of this knot is $12+\log (\mathrm{O} / \mathrm{H})=8.1$, which is 0.4 dex lower than that found in nearby complexes. We suggest that this region actually is an independent dwarf galaxy or its remnant.

Although the XUV complexes are very likely induced by the NGC 1512/1510 interaction, they cannot be strictly defined as TDGs, as they have not been formed from material stripped from the galaxies, but from the diffuse gas already existing at large galactocentric radii. We propose to define these UV-bright, young, relatively low-metallicity, gas-rich entities to be "tidally-induced star-forming clusters" (TSFCs) in the galaxy outskirts.

\section{Conclusions of the multiwavelength analysis of NGC $1512 / 1510$}

Our data suggest that the gas in the outer regions of NGC 1512 already had a metallicity of $12+\log (\mathrm{O} / \mathrm{H}) \sim 8.1$ about $400 \mathrm{Myr}$ ago, before the interaction with NGC 1510 started. The metals within the diffuse H I gas are very likely not coming from the inner regions of NGC 1512, but probably from material accreted during minor merger events during the life of the galaxy. We indeed found a probable remnant of an independent dwarf galaxy and three knots which may also be independent systems, all of them having $12+\log (\mathrm{O} / \mathrm{H}) \lesssim 8.1$. This hypothesis agrees with the finding around other nearby large galaxies (e.g. Bresolin et al. 2009; Bresolin, Kennicutt \& Ryan-Weber 2012), as well as constraints chemical and dynamical models of galaxy evolution.

\section{References}

Bresolin, F., et al. 2009, ApJ, 700, 309

Bresolin, F., Kennicutt, R. C., \& Ryan-Weber, E., 2012, ApJ, 750, 122

Gil de Paz, A., et al. 2005, ApJL, 627, L29

Koribalski, B. S., 2008, The Local Volume H I Survey, Koribalski B. S., Jerjen H., eds. p. 41

Koribalski, B. S. \& López-Sánchez, Á. R., 2009, MNRAS, 400,1749

Thilker, D. A., et al. 2005, ApJL, 619, L79 